\title{
Biliary Peritonitis Following the Removal of a T-tube
}

Sqn Ldr S D H Holt
MA, FRCS, RAF

Senior Specialist in Surgery

Princess Mary's Royal Air Force Hospital, Halton, Aylesbury, Bucks

\section{Gp Capt I B Tiwari}

FRCS, RAF

Consultant Surgeon

Royal Air Force Hospital, Ely, Cambs

\section{Sqn Ldr G P Howell}

FRCS, RAF

Specialist in Surgery

Royal Air Force Hospital, Akrotiri, Cyprus

\section{Introduction}

The removal of a $\mathrm{T}$-tube following exploration of a common bile duct (CBD) rarely results in complications. However, we wish to report a case of biliary peritonitis following the removal of a latex rubber T-tube.

\section{Case Report}

An otherwise fit 28 year old female presented with a four year history of biliary colic. Cholecystectomy was carried out through an upper right paramedian incision. The per-operative cholangiogram showed a single small calculus at the lower end of the CBD. The stone was removed with some difficulty through a longitudinal choledochotomy. A 16FG latex "Kehr T-tube" was inserted and the CBD closed with interrupted chromic catgut taking care not to include the tube in the suture. The long limb of the tube was brought out to the right of the incision.

The patient made an uneventful post operative recovery and a $\mathrm{T}$-tube cholangiogram at 10 days showed free flow into the duodenum and no evidence of stones. The T-tube was removed without difficulty, but immediately the patient began to complain of deep right upper quadrant pain. Over the next 12 hours the pain spread to the right iliac fossa and then became generalised. On examination the patient was pyrexial (38.0) with a tachycardia (100) and demonstrated generalised tenderness, guarding and rebound.

At emergency laparotomy $450 \mathrm{ml}$ of lightly blood stained bile was removed from the peritoneal cavity. The leak appeared to be from the site of the choledochotomy rather than any other part of the well formed T-tube track. Peritoneal lavage was performed and suction drains placed in the vicinity of the leak and in the right paracolic gutter.

The patient subsequently made an uncomplicated recovery.

\section{Discussion}

Three other cases of biliary peritonitis following the removal of latex rubber T-tubes have been reported ${ }^{1,2}$, although the problem is certainly commoner than this would suggest. Other materials such as polyvinyt chloride and silicon rubber have been found unsuitablec⿱ because removal tends to be followed by leakage , $^{3,4}$.

We wish to make two observations about this case. Firstly, although the use of large T-tubes facilitates the subsequent removal of any retained stones using to steerable catheter inserted down the track, it seem likely that the large size of the tube in relation to the duct used in this case was the main cause of the subsequent leak.

Secondly, treatment of this complication is contentious. Conservative management has been used but may predispose to the development of subphrenic abscess ${ }^{3}$. Reinsertion of the T-tube at emergency laparotomy is an alternative treatment which has given satisfactory results ${ }^{3}$, but this may prove technically difficult if dense adhesions have formed. Laparotomy and adequate drainage from the region of the leak proved easy and successful in this case, and we think this procedure should be considered first when treating biliary peritonitis which occurs following the removal of a $T$ tube.

\section{REFERENCES}

1. Michotey G, et al. Les complications du drain de kehr. A propos de quatre observations. Ann Chir 1981; 35: $351-355$.

2. Osbourne J C. Bile peritonitis after T-tube removal. Can J Surg 1971; 14: 241-242.

3. WINSTONEN E. et al. Biliary peritonitis: a hazard of polyvinyl chloride T-tubes. Lancet 1965; 1: 843-844.

4. Apalakis A. An experimental evaluation of the types of material used for bile duct drainage tubes. Br J Surg 1976 ; 63: $440-445$. 


\title{
Hospital Acquired Native Valve Endocarditis Caused by Acinetobacter calcoaceticus and Treated with Imipenem/Cilastin
}

\author{
Major N S Cumberland \\ MRCPath RAMC \\ Senior Specialist Pathology* \\ Captain K P Jones \\ $\mathrm{MB}, \mathrm{ChB}, \mathrm{RAMC}$ \\ Queen Elizabeth Military Hospital, Woolwich, London SE18
}

SUMMARY: A case of hospital acquired endocarditis due to Acinetobacter calcoaceticus in a severely burned patient is $\vec{\odot}$ presented. Both aortic and mitral native valves were affected and the organism was resistant to most antimicrobial agents.

\section{Introduction}

Acinetobacter calcoaceticus, a Gram-negative nonfermentative aerobic cocco-bacillus, is a frequent and often persistant coloniser of the hands of hospital personnel and of the skin and urine of in-patients ${ }^{1}$. It is isolated from burn wounds ${ }^{2}$ and infrequently as a culture contaminant from blood cultures ${ }^{3}$ and may cause a wide variety of infections ${ }^{4,5}$.

Acinetobacter is an uncommon pathogen, usually affecting the seriously ill and compromised patient, causing $1.4 \%$ of hospital acquired infections ${ }^{4}$.

Few well documented cases of endocarditis due to $A$. calcoaceticus have been reported ${ }^{5-8}$, and, to our knowledge none from the UK. We present a case of hospital acquired Acinetobacter endocarditis in an extensively burned patient.

\section{Case Report}

In a bomb explosion, a 27 year old soldier sustained $40 \%$ burns involving limbs and trunk, and injuries to his left leg which required below knee amputation. During his first 12 weeks in hospital he developed continual pyrexia, hypoproteinaemia, lost $30 \mathrm{kgs}$ and underwent seven procedures for excision of slough and skin grafting. Cloxacillin and ampicillin were started on admission and continued for 25 days. Netilmicin and piperacillin were given from days 22 to 28 for clinical septicaemia but with negative blood cultures.

A. calcoaceticus was isolated in pure culture from a catheter specimen of urine on day 35, but disappeared spontaneously after subsequent change of catheter. Thereafter, his clinical condition and burn wounds improved and no antibiotics were given until day 76 when he received cephalexin for 12 days, followed by piperacillin from day 88 to 94 for purulent graft sites, from which two coliforms and Pseudomonas aeruginosa were isolated. On day 84 he underwent further skin grafting, and on day 87 insertion of a venous catheter. A. calcoaceticus was isolated from a single blood culture, taken on day 87, after 7 days incubation. During week 13 his condition deteriorated, with anaemia, persistant tachycardia and pyrexia, and a systolic murmur was heard for the first time. There was no additional evidence of endocarditis. One of two blood cultures $\vec{\omega}$ taken on day 95 grew $A$. calcoaceticus after 5 days incubation; Klebsiella sp was isolated from his urine (day 96) and cephalexin was given again. His condition $\propto$ improved temporarily after blood transfusion, but 을 eleven days later (day 107) he deteriorated sudden with tachycardia, tachypnoea, toxaemia and confusiof Aortic systolic and diastolic murmers were presene of Ampicillin and gentamicin were started. Within 2 das he had developed severe aortic regurgitation and congestive cardiac failure. The electrocardiogram $\varnothing$ showed atrio-ventricular conduction defects and.v echocardiograms indicated vegetations on the aortic and mitral valves.

A. calcoaceticus was isolated from blood cultures taken on days 107 and 109, and from his urine. At operation on day 111 the aortic valve was found to be destroyed, with large vegetations on the aortic root and septum. There were multiple abscess cavities around the annulus and two septal abscesses. The aortic valve was replaced with a prosthetic bovine pericardial valve, and vegetations on the anterior mitral leaflet removed and perforations repaired.

A heavy pure growth of $A$. calcoaceticus was obtained from the aortic valve vegetations. On the 10th day after operation he developed acute pulmonary oedema with worsening mitral valve regurgitation. Echocardiography showed a vegetation on the mitral valve; the next day this valve was replaced and a pure growth of $A$. calcoaceticus was obtained from the vegetation.

A variety of antibiotic combinations (see Fig 1) was given until detailed susceptibility tests were available 윽 when Imipenem plus cilastin (Merck, Sharp and Dohme $>$ Research Laboratories) was started on day 125 and continued for 21 days. He was apyrexial within 5 days of $N$ starting this agent but his cardiac function continued to deteriorate and permanent pacing was required. He ${ }^{\circ}$ developed irreversible left ventricular failure and died $\mathcal{N}^{2}$ on day 155. At autopsy, the prosthetic aortic and mitralo

* Now Consultant Pathologist and Commanding Officer, David Bruce Laboratories, East Everleigh, Nr Marlborough, Wilts. 


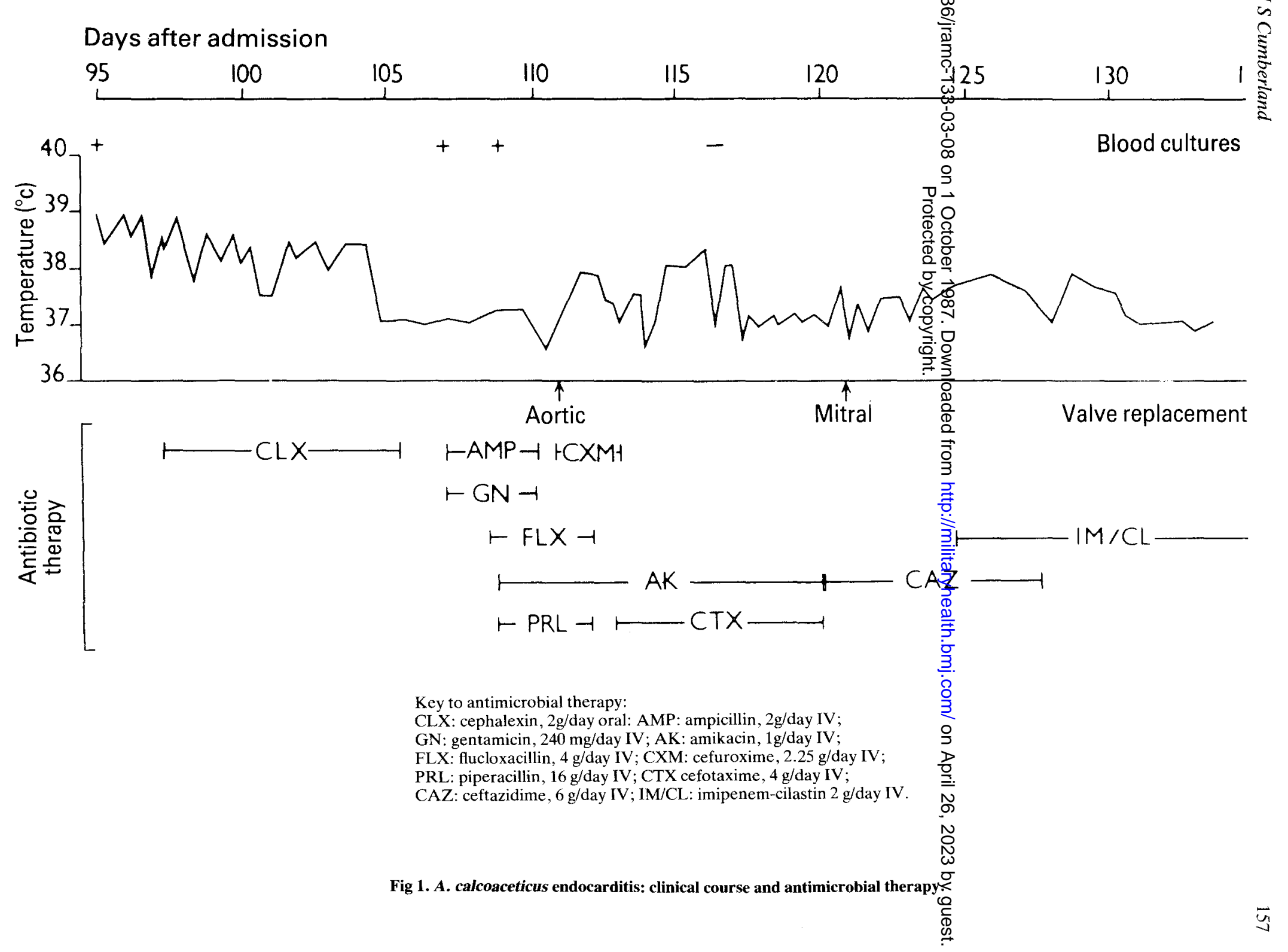


valves and native pulmonary and tricuspid valves were intact, free from vegetations and sterile on culture.

\section{Microbiology}

All isolates of $A$. calcoaceticus exhibited similar antimicrobial susceptibilities as follows: Imipenem, Minimum Inhibitory Concentration (MIC) and Minimum Bactericidal Concentration (MBC) $0.25 \mathrm{mg} /$ $\mathrm{L}$; tobramycin, MIC $2 \mathrm{mg} / \mathrm{L}$; amikacin, MIC $4 \mathrm{mg} / \mathrm{L}$; MBC 4-8 $\mathrm{mg} / \mathrm{L} ;$ gentamicin, $\mathrm{MIC}>128 \mathrm{mg} / \mathrm{L}$; ampicillin, MIC $>128 \mathrm{mg} / \mathrm{L} ;$ piperacillin, $\mathrm{MIC}>256$ $\mathrm{mg} / \mathrm{L}$; carbenicillin, MIC $2048 \mathrm{mg} / \mathrm{L}$; cephalexin, MIC $>128 \mathrm{mg} / \mathrm{L}$; cefotaxime, MIC $16 \mathrm{mg} / \mathrm{L}$; cefuroxime, MIC $32 \mathrm{mg} / \mathrm{L}$; ceftazidime, MIC 4-8 $\mathrm{mg} / \mathrm{L}$ and MBC $8 \mathrm{mg} / \mathrm{L}$ and trimethoprim/sulphamethoxazole, MIC 6.4/ $128 \mathrm{mg} / \mathrm{L}$.

\section{Comment}

Acute bacterial endocarditis arising from sepsis and bacteraemia is a recognised though uncommon complication of burns ${ }^{9}$.

Acinetobacter endocarditis may involve both normal and diseased native valves, or prosthetic valves or the congenitally abnormal heart ${ }^{5-8}$. In our patient there was no evidence of pre-existing heart disease.

Acinetobacter bacteraemia most commonly results from colonised or infected intravenous catheters, but it may also occur with other infections including those in burns $^{2,5}$. Antecedent broad spectrum antibiotics have invariably been given ${ }^{2,5,6}$. Before endocarditis developed $A$. calcoaceticus was isolated only from urine in our patient and early positive blood cultures (days 87 and 95) were considered not to represent bacteraemia. Acinetobacter was specifically sought, but was not isolated, from his burn wounds (although the sub-eschar space was not sampled), intravenous catheters and sputum. He had, however, been in hospital for 12 weeks, and undergone multiple surgical procedures to colonised wounds, repeated intravenous and urinary catheterisation, and had received prolonged and varied broad spectrum antibiotics. These factors, together with the compromised physiological and immunological functions following extensive burn injury, will predispose a patient to bacteraemia with organisms of low pathogenicity ${ }^{2,5}$.
In common with previous reports ${ }^{2,4,5}$, this strain of $A$. calcoaceticus was resistant to most antibiotics, including gentamicin and all cephalosporins. Imipenem, a carbapenem B-lactam with a very broad antimicrobialo spectrum, was most active in vitro and appears to have eradicated the infection, but it is difficult to assess the role of any antibiotic when valve replacement is undertaken.

\section{Acknowledgements}

The patient was treated in the Queen Elizabeth Military Hospital, Woolwich and Guy's Hospital, London. We would like to thank Mr P B Deverall for. permission to publish this report and Dr N A Simmons $\overrightarrow{\vec{\omega}}$ (Guy's Hospital) and Dr S J Eykyn (St Thomas' Hospital) for their help and advice.

\section{REFERENCES}

1. FRENCH G L et al. A hospital outbreak of antibioticresistant Acinetobacter anitratus: epidemiology and control. J Hosp Inf 1980; 1: 125-131.

2. Green A R and Milling M A P. Infection with Acineto-o bacter in a burns unit. Burns Incl Therm Inj 1983; g. 292-294.

3. Al-Khoja M S and Darrell J H. The skin as the source $\mathrm{d} f$ Acinetobacter and Moraxella species occurring in blood cultures. J Clin Pathol 1979; 32: 497-499.

4. LARSON E. A decade of nosocomial Acinetobacter. An Infection Control 1984; 12: 14-18.

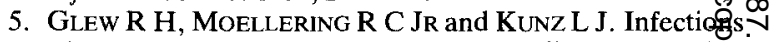
with Acinetobacter calcoaceticus (Herellea vaginicol Clinical and Laboratory studies. Medicine (Baltimo 1977; 56: 79-97.

6. Cohen $P$ S, Maguire $\mathbf{J} \mathbf{H}$ and Weinstein $L$. Infective endocarditis caused by Gram-negative bacteria: A review음 of the literature 1945-1977. Prog Cardiovasc Dis 1980; 22; 205-242.

7. Rao K N A, Kotian M and Prabhu S G S. Infective endocarditis due to Acinetobacter calcoaceticus. J. Postgrad Med 1980; 26: 186-191.

8. Pal P B, Sujatha V and Kale V V. Acinetobacter calcoaceticus causing subacute bacterial endocarditis. Lancet 1981; II: 313 .

9. Baskin $\mathbf{T}$ W, Rosenthal $\mathbf{A}$ and Pruitt B A. Acute bacterial endocarditis: A silent source of sepsis in the burn $\frac{D}{\Phi}$ patient. Ann. Surg 1976; 184: 618-621. 